\title{
Principales medidas en epidemiología
}

\author{
Alejandra Moreno-Altamirano, C.D., M. en C., ${ }^{(1)}$ Sergio López-Moreno, M.C., ${ }^{(2)}$ \\ Alexánder Corcho-Berdugo, M.C.(2)
}

\section{Concepto de medición, variables y escalas}

U na vez que se ha identificado un problema científico y se ha aventurado una explicación hipotética, es necesario someterla a prueba. Para contrastar la hipótesis se requiere descomponerla en un conjunto suficientemente pequeño de variables susceptibles de ser evaluadas empíricamente. Si los procedimientos empíricos no refutan la hipótesis planteada ésta se acepta como probablemente verdadera. En pocas palabras, este es el camino que el científico sigue más frecuentemente al realizar su trabajo. Dado que en la mayoría de los casos es necesario medir las variables durante la contrastación empírica de la hipótesis, la medición resulta un procedimiento indispensable en la práctica científica.

En epidemiología, el proceso de investigación es similar al utilizado en el resto de las ciencias. Cuando se investiga la salud de la población también se proponen una o varias explicaciones hipotéticas que posteriormente son sometidas a contrastación empírica. En este proceso, los conceptos de medición y de variable resultan fundamentales.

\section{Concepto de variable}

La función de las variables consiste en proporcionar información asequible para descomponer la hipótesis planteada en sus elementos más simples. Las variables pueden definirse como aquellos atributos o características de los eventos, de las personas o de los grupos de estudio que cambian de una situación a otra o de un tiempo a otro y que, por lo tanto, pueden tomar diversos valores. Para su estudio es necesario medirlas en el objeto investigado, y es en el marco del problema y de las hipótesis planteadas donde adquieren el carácter de variables.

De acuerdo con la relación que guardan unas con otras, las variables se clasifican en independientes (o variables explicativas) y dependientes (o variables respuesta). Cuando se supone que una variable produce un cambio en otra, se considera a la primera como independiente (o causa) y a la segunda como dependiente (o efecto). En los estudios epidemiológicos la enfermedad o evento es por lo general la variable dependiente y los factores que determinan su aparición, magnitud y distribución son las variables independientes, o exposición. No obstante, el concepto de dependencia e independencia es contextual, es decir, obedece al modelo teórico planteado. Una vez que se han identificado las variables el investigador debe definirlas de manera operativa, especificando el método y la escala con las cuales llevará a cabo su medición.

El uso de variables permite a la epidemiología la elaboración de modelos descriptivos, explicativos y predictivos sobre la dinámica de la salud poblacional. En los modelos más sencillos (por ejemplo, en los modelos en los que se considera una sola exposición y un solo daño o evento) las variables generalmente se expresan en tablas simples de dos categorías mutuamente excluyentes (llamadas dicotómicas), representadas por la ausencia y la presencia de la exposición y la

(1) Departamento de Salud Pública, Facultad de Medicina, Universidad N acional Autónoma de México.

(2) Dirección de Políticas y Planeación, Centro de Investigación en Sistemas de Salud, Instituto N acional de Salud Pública, México. 
ausencia y la presencia del evento. Al combinar ambas categorías se forma una tabla con dos filas y dos columnas, conocida como tabla tetracórica o tabla de 2 por 2. Cuando, en cambio, existen más de dos categorías de exposición, o varias formas de clasificar el evento, esta relación se expresa en tablas de varias columnas y varias celdas. En este texto se analizará la elaboración de medidas epidemiológicas basadas en categorías dicotómicas y el uso de tablas de $2 \times 2$.

\section{Concepto de medición}

La medición consiste en asignar un número o una calificación a alguna propiedad específica de un individuo, una población o un evento usando ciertas reglas. No obstante, la medición es un proceso de abstracción. En términos estrictos no se mide al individuo sino cierta característica suya, abstrayéndola de otras propiedades. Uno no mide al niño sino que obtiene información sobre su estatura o su peso. Además, lo que se hace es comparar el atributo medido en otros individuos (o en el mismo individuo en otro momento), con el fin de evaluar sus cambios en el tiempo o cuando se presenta en condiciones distintas de las originales.

Para medir es necesario seguir un proceso que consiste, en breves palabras, en el paso de una entidad teórica a una escala conceptual y, posteriormente, a una escala operativa.

En general, los pasos que se siguen durante la medición son los siguientes: a) se delimita la parte del evento que se medirá, b) se selecciona la escala con la que se medirá, c) se compara el atributo medido con la escala y, d) finalmente, se emite un juicio de valor acerca de los resultados de la comparación. Para medir el crecimiento de un menor, por ejemplo, primero se selecciona la variable a medir (la edad, el peso, la talla); luego se seleccionan las escalas de medición (meses cumplidos, centímetros, gramos); inmediatamente después se comparan los atributos con las escalas seleccionadas (un mes de edad, $60 \mathrm{~cm}$ de talla, 4500 gramos de peso) y, por último, se emite un juicio de valor, que resume la comparación entre las magnitudes encontradas y los criterios de salud aceptados como válidos en ese momento. Como resultado, el infante se califica como bien nutrido, desnutrido o sobrenutrido.

Como se puede notar, la medición es un proceso instrumental sólo en apariencia, ya que la selección de la parte que se medirá, de la escala de medición y de los criterios de salud que se usarán como elementos de juicio deben ser resultado de un proceso de decisión teórica. En otras palabras, sólo puede medirse lo que antes se ha concebido teóricamente. La medición, sin embargo, nos permite alcanzar un alto grado de objetividad al usar los instrumentos, escalas y criterios aceptados como válidos por la mayor parte de la comunidad científica.

\section{Principales escalas de medición}

Las escalas se clasifican en cualitativas (nominal y ordinal) y cuantitativas (de intervalo y de razón). Un requisito indispensable en todas las escalas es que las categorías deben ser exhaustivas y mutuamente excluyentes. En otras palabras, debe existir una categoría para cada caso que se presente y cada caso debe poder colocarse en una sola categoría.

\section{Escala nominal}

La medición de carácter nominal consiste simplemente en clasificar las observaciones en categorías diferentes con base en la presencia o ausencia de cierta cualidad. De acuerdo con el número de categorías resultantes, las variables se clasifican en dicotómicas (dos categorías) o politómicas (más de dos categorías). En las escalas nominales no es posible establecer un orden de grado como mejor o peor, superior o inferior, o más o menos. La asignación de códigos numéricos a las categorías se hace con el único fin de diferenciar unas de otras y no tienen interpretación en lo que se refiere al orden o magnitud del atributo. Como ejemplos de este tipo de medición en la investigación epidemiológica se pueden mencionar el sexo (masculino " 0 ", femenino " 1 "), el estado civil (soltero, casado, viudo, divorciado), la exposición o no a un factor $X$, y el lugar de nacimiento, entre otras.

\section{Escala ordinal}

En contraste con las escalas nominales, en este tipo de medición las observaciones se clasifican y ordenan por categorías según el grado en que los objetos o eventos poseen una determinada característica. Por ejemplo, se puede clasificar a las personas con respecto al grado de una enfermedad en leve, moderado o severo. Si se llega a utilizar números en este tipo de escalas su única significación consiste en indicar la posición de las distintas categorías de la serie y no la magnitud de la diferencia entre las categorías. Para la variable antes mencionada, por ejemplo, sabemos que existe una diferencia de grado entre leve y severo, pero no es posible establecer con exactitud la magnitud de la diferencia en las enfermedades de una $u$ otra personas. 


\section{Escala de intervalo}

Esta es una escala de tipo cuantitativo en la que, además de ordenar las observaciones por categorías del atributo, se puede medir la magnitud de la distancia relativa entre las categorías. Esta escala, sin embargo, no proporciona información sobre la magnitud absoluta del atributo medido. Por ejemplo, se puede obtener una escala de intervalo para la altura de las personas de un grupo si, en lugar de medirlas directamente, se mide la altura de cada persona con respecto a la altura promedio. En este caso, el valor cero es arbitrario y los valores asignados a la altura no expresan su magnitud absoluta. Esta es la característica distintiva de las escalas de intervalo en comparación con las de razón.

El ejemplo más conocido de las escalas de intervalo es la escala de Celsius para medir la temperatura, en la que por convención el grado cero corresponde al punto de congelación del agua y donde, por lo tanto, la razón entre dos objetos con temperaturas de 10 y 20 grados no indica que uno de ellos sea realmente dos veces más caliente (o más frío) que el otro. En ciencias de la salud, un buen ejemplo de este tipo de escalas es la utilizada para medir el coeficiente intelectual.

Escalas de razón

Esta escala tiene la cualidad de que el cero sí indica la ausencia del atributo y, por lo tanto, la razón entre dos números de la escala es igual a la relación real existente entre las características de los objetos medidos. En otras palabras, cuando decimos que un objeto pesa $8 \mathrm{~kg}$ estamos también diciendo que pesa el doble que otro cuyo peso es de $4 \mathrm{~kg}$, y que un avión que viaja a $600 \mathrm{~km}$ por hora tardará en llegar a su destino la mitad del tiempo que tardaría si viajara a $300 \mathrm{~km}$ por hora. Muchas características biofísicas y químicas que pueden ser medidas en las unidades convencionalmente aceptadas (metros, gramos, micras, $\mathrm{mol} / \mathrm{kg}, \mathrm{mg} / \mathrm{dl}$, etc.) son ejemplos de mediciones que corresponden a este tipo de escala. En materia de investigación social y de salud, el ingreso económico y la concentración de plomo en sangre son buenos ejemplos de este tipo de escalas.

\section{Cálculo de proporciones, tasas y razones}

Un rasgo característico de la contrastación en los estudios epidemiológicos es que las relaciones causales postuladas entre las variables se traducen en términos probabilísticos. Es decir, se trata de establecer si la mayor o menor probabilidad de que un evento ocurra se debe precisamente a los factores que se sospecha intervienen en su génesis y no al azar. Para cumplir con este objetivo, la investigación epidemiológica se basa en la construcción de tres tipos de medidas: a) de frecuencia; b) de asociación o efecto, y c) de impacto potencial. La construcción de estas medidas se realiza por medio de operaciones aritméticas simples y de los instrumentos matemáticos conocidos como razones, proporciones y tasas. Antes de abordar las medidas utilizadas en los estudios epidemiológicos repasaremos brevemente estos tres conceptos.

\section{Proporciones}

Las proporciones son medidas que expresan la frecuencia con la que ocurre un evento en relación con la población total en la cual éste puede ocurrir. Esta medida se calcula dividiendo el número de eventos ocurridos entre la población en la que ocurrieron. Como cada elemento de la población puede contribuir únicamente con un evento es lógico que al ser el numerador (el volumen de eventos) una parte del denominador (población en la que se presentaron los eventos) aquel nunca pueda ser más grande que éste. Esta es la razón por la que el resultado nunca pueda ser mayor que la unidad y oscile siempre entre cero y uno.

Por ejemplo, si en un año se presentan tres muertes en una población compuesta por 100 personas, la proporción anual de muertes en esa población será:

$P=\frac{3 \text { muertes }}{100 \text { personas }}=0.03$

A menudo las proporciones se expresan en forma de porcentaje, y en tal caso los resultados oscilan entre cero y 100. En el ejemplo anterior, la proporción anual de muertes en la población sería de 3 por 100 , o de $3 \%$. Nótese, asimismo, que el denominador no incluye el tiempo. Las proporciones expresan únicamente la relación que existe entre el número de veces en las que se presenta un evento y el número total de ocasiones en las que se pudo presentar.

\section{Tasas}

Las tasas expresan la dinámica de un suceso en una población a lo largo del tiempo. Se pueden definir como la magnitud del cambio de una variable (enfermedad o muerte) por unidad de cambio de otra (usualmente el tiempo) en relación con el tamaño de la población que se encuentra en riesgo de experimentar el suceso. 
En las tasas, el numerador expresa el número de eventos acaecidos durante un periodo en un número determinado de sujetos observados.

A diferencia de una proporción el denominador de una tasa no expresa el número de sujetos en observación sino el tiempo durante el cual tales sujetos estuvieron en riesgo de sufrir el evento. La unidad de medida empleada se conoce como tiempo-persona de seguimiento. Por ejemplo, la observación de 100 individuos libres del evento durante un año corresponde a 100 años-persona de seguimiento; de manera similar, 10 sujetos observados durante diez años corresponden a 100 años-persona.

Dado que el periodo entre el inicio de la observación y el momento en que aparece un evento puede variar de un individuo a otro, el denominador de la tasa se estima a partir de la suma de los periodos de todos los individuos. Las unidades de tiempo pueden ser horas, días, meses o años, dependiendo de la naturaleza del evento que se estudia.

El cálculo de tasas se realiza dividiendo el total de eventos ocurridos en un periodo dado en una población entre el tiempo-persona total (es decir, la suma de los periodos individuales libres de la enfermedad) en el que los sujetos estuvieron en riesgo de presentar el evento. Las tasas se expresan multiplicando el resultado obtenido por una potencia de 10, con el fin de permitir rápidamente su comparación con otras tasas.

\footnotetext{
número de eventos ocurridos en

Tasa $=\frac{\text { una población en un periodo } t}{\text { sumatoria de los periodos durante }} \times$ una potencia de 10

los cuales los sujetos de la población libres

del evento estuvieron expuestos al riesgo

de presentarlo en el mismo periodo
}

\section{Razones}

Las razones pueden definirse como magnitudes que expresan la relación aritmética existente entre dos eventos en una misma población, o un solo evento en dos poblaciones. En el primer caso, un ejemplo es la razón de residencia hombre: mujer en una misma población. Si en una localidad residen 5000 hombres y 4000 mujeres se dice que, en ese lugar, la razón de residencia hombre:mujer es de 1:0.8 (se lee 1 a 0.8), lo que significa que por cada hombre residen ahí 0.8 mujeres. Esta cantidad se obtiene como sigue:

Razón hombre: mujer $=\frac{4000}{5000}=0.8$
En este caso, también se podría decir que la razón hombre:mujer es de 10:8, pues esta expresión aritmética es igual a la primera (1:0.8).

En el segundo ejemplo se encuentran casos como la razón de tasas de mortalidad por causa específica (por ejemplo, por diarreas) en dos comunidades. En este caso, la razón expresaría la relación cuantitativa que existe entre la tasa de mortalidad secundaria a diarreas registrada en la primera ciudad y la tasa de mortalidad secundaria a diarreas registrada en la segunda. La razón obtenida expresa la magnitud relativa con la que se presenta este evento en cada población. Si la tasa de mortalidad por diarreas en la primera ciudad es de 50 por 1000 y en la segunda de 25 por 1000 la razón de tasas entre ambas ciudades sería:

$\mathrm{RTM}=\frac{\text { tasa de mortalidad en la ciudad B }}{\text { tasa de mortalidad en la ciudad A }}=\frac{50 \times 1000}{25 \times 1000}=2.0$

Donde RTM es la razón de tasas de mortalidad (en este caso, por diarreas) entre las ciudades A y B. El resultado se expresa como una razón de 1:2, lo que significa que por cada caso en la ciudad A hay 2 en la ciudad B.

\section{Medidas de frecuencia}

El paso inicial de toda investigación epidemiológica es medir la frecuencia de los eventos de salud con el fin de hacer comparaciones entre distintas poblaciones o en la misma población a través del tiempo. No obstante, dado que el número absoluto de eventos depende en gran medida del tamaño de la población en la que se investiga, estas comparaciones no se pueden realizar utilizando cifras de frecuencia absoluta (o número absoluto de eventos).

Por ejemplo, si en dos diferentes poblaciones se presentan 100 y 200 casos de cáncer cervicouterino, respectivamente, se podría pensar que en el segundo grupo la magnitud del problema es del doble que en el primero. Sin embargo, esta interpretación sería incorrecta si el segundo grupo tuviera el doble de tamaño que el primero, ya que la diferencia en el número de casos podría deberse simplemente al mayor tamaño de la segunda población y no a la presencia de un factor de riesgo extraordinario. Aunque la frecuencia absoluta cambie la magnitud del problema puede ser la misma.

En consecuencia, para comparar adecuadamente la frecuencia de los eventos de salud es necesario construir una medida que sea independiente del tamaño 
de la población en la que se realiza la medición. Este tipo de medidas, denominadas medidas de frecuencia relativa, se obtiene, en general, relacionando el número de casos (numerador) con el número total de individuos que componen la población (denominador). El cálculo correcto de estas medidas requiere que se especifique claramente qué constituye el numerador y el denominador. Es evidente, por ejemplo, que los varones no deben ser incluidos en el denominador durante el cálculo de la frecuencia relativa de carcinoma del cérvix.

La parte de la población que es susceptible a una enfermedad se denomina población en riesgo. Así, por ejemplo, los accidentes laborales sólo afectan a las personas que trabajan, por lo que la población en riesgo es la población trabajadora. $\mathrm{Si}$, en cambio, queremos investigar el efecto de un contaminante generado por una fábrica podríamos ampliar el denominador a toda la población expuesta al mismo, sea o no trabajadora.

Las medidas de frecuencia más usadas en epidemiología se refieren a la medición de la mortalidad o la morbilidad en una población. La mortalidad es útil para estudiar enfermedades que provocan la muerte, especialmente cuando su letalidad es importante. Empero, cuando la letalidad es baja y, en consecuencia, la frecuencia con la que se presenta una enfermedad no puede analizarse adecuadamente con los datos de mortalidad, la morbilidad se convierte en la medida epidemiológica de mayor importancia.

En ocasiones, la morbilidad también puede servir para explicar las tendencias de la mortalidad, ya que los cambios en la mortalidad pueden ser secundarios a cambios ocurridos antes en la morbilidad o, por el contrario, las tendencias en la mortalidad pueden explicar los cambios en los patrones de morbilidad cuando, por ejemplo, la disminución en la mortalidad infantil explica los aumentos aparentes en el volumen de enfermedades en otras edades. Por ambas razones, el análisis de las condiciones de salud de las poblaciones se basa siempre en los cambios observados en las medidas de mortalidad y morbilidad.

Las principales fuentes de información de morbilidad son los datos hospitalarios y los registros de enfermedad. Sin embargo, debido a las limitaciones de estos registros, los estudios epidemiológicos se basan en información obtenida mediante métodos de detección especialmente diseñados para ello. A continuación se presenta un resumen de los elementos más importantes de las medidas de mortalidad y morbilidad.

\section{Medidas de mortalidad}

El concepto de mortalidad expresa la magnitud con la que se presenta la muerte en una población en un momento determinado. A diferencia de los conceptos de muerte y defunción que reflejan la pérdida de la vida biológica individual, la mortalidad es una categoría de naturaleza estrictamente poblacional. En consecuencia, la mortalidad expresa la dinámica de las muertes acaecidas en las poblaciones a través del tiempo y el espacio, y sólo permite comparaciones en este nivel de análisis. La mortalidad puede estimarse para todos o algunos grupos de edad, para uno o ambos sexos y para una, varias o todas las enfermedades. La mortalidad se clasifica de la siguiente manera: a) general y $b$ ) específica.

\section{Mortalidad general}

La mortalidad general es el volumen de muertes ocurridas por todas las causas de enfermedad, en todos los grupos de edad y para ambos sexos. La mortalidad general, que comúnmente se expresa en forma de tasa, puede ser cruda o ajustada, de acuerdo con el tratamiento estadístico que reciba.

La mortalidad cruda expresa la relación que existe entre el volumen de muertes ocurridas en un periodo dado y el tamaño de la población en la que éstas se presentaron; la mortalidad ajustada (o estandarizada) expresa esta relación pero considera las posibles diferencias en la estructura por edad, sexo, etcétera, de las poblaciones analizadas, lo que permite hacer comparaciones entre éstas. En este caso, las tasas se reportan como tasas ajustadas o estandarizadas. La tasa cruda de mortalidad se calcula de acuerdo con la siguiente fórmula:

$$
\text { Tasa mortalidad general }=\frac{\begin{array}{c}
\text { número de muertes en } \\
\text { el periodo } t
\end{array}}{\begin{array}{c}
\text { población total promedio } \\
\text { en el mismo periodo }
\end{array}}(\times 10 \mathrm{n})
$$

\section{Mortalidad específica}

Cuando existen razones para suponer que la mortalidad puede variar entre los distintos subgrupos de la población ésta se divide para su estudio. Cada una de las medidas obtenidas de esta manera adopta su nombre según la fracción poblacional que se reporte. Por ejemplo, si las tasas de mortalidad se calculan para los diferentes grupos de edad, serán denominadas tasas de mortalidad por edad. De la misma manera pueden calcularse la mortalidad por sexo, por causa específica, etcétera.

En algunos casos pueden calcularse combinaciones de varias fracciones poblacionales, y cuando es así, se especifican los grupos considerados (por ejemplo, mortalidad femenina en edad reproductiva). Las ta- 
sas de mortalidad específica por edad y sexo se calculan de la siguiente forma:

total de muertes en un grupo de edad

y sexo específicos de la población durante

$\mathrm{TME}=\frac{\text { un periodo dado }}{\begin{array}{c}\text { población total estimada del mismo grupo } \\ \text { de edad y sexo en el mismo periodo }\end{array}}\left(\begin{array}{l}x 10 \mathrm{n}) \\ \text {. }\end{array}\right.$

Donde TME es la tasa de mortalidad específica para esa edad y sexo.

Tasa de letalidad. La letalidad es una medida de la gravedad de una enfermedad considerada desde el punto de vista poblacional, y se define como la proporción de casos de una enfermedad que resultan mortales con respecto al total de casos en un periodo especificado. La medida indica la importancia de la enfermedad en términos de su capacidad para producir la muerte y se calcula de la manera siguiente:

$$
\begin{gathered}
\text { número de muertes por una enfermedad } \\
\text { Letalidad }(\%)=\frac{\text { en un periodo determinado }}{\text { número de casos diagnosticados de }} \times 100 \\
\text { la misma enfermedad en el mismo periodo }
\end{gathered}
$$

La letalidad, en sentido estricto, es una proporción ya que expresa el número de defunciones entre el número de casos del cual las defunciones forman parte. No obstante, generalmente se expresa como tasa de letalidad y se reporta como el porcentaje de muertes de una causa específica con respecto al total de enfermos de esa causa.

\section{Medidas de morbilidad}

La enfermedad puede medirse en términos de prevalencia o de incidencia. La prevalencia se refiere al número de individuos que, en relación con la población total, padecen una enfermedad determinada en un momento específico. Debido a que un individuo sólo puede encontrarse sano o enfermo con respecto a cualquier enfermedad, la prevalencia representa la probabilidad de que un individuo sea un caso de dicha enfermedad en un momento específico.

La incidencia, por su parte, expresa el volumen de casos nuevos que aparecen en un periodo determinado, así como la velocidad con la que lo hacen; es decir, expresa la probabilidad y la velocidad con la que los individuos de una población determinada desarrollarán una enfermedad durante cierto periodo.

\section{Prevalencia}

La prevalencia es una proporción que indica la frecuencia de un evento. En general, se define como la proporción de la población que padece la enfermedad en estudio en un momento dado, y se denomina únicamente como prevalencia $(p)$. Como todas las proporciones, no tiene dimensiones y nunca puede tomar valores menores de 0 o mayores de 1 . A menudo, se expresa como casos por 1000 o por 100 habitantes.

En la construcción de esta medida no siempre se conoce en forma precisa la población expuesta al riesgo y, por lo general, se utiliza sólo una aproximación de la población total del área estudiada. Si los datos se han recogido en un momento o punto temporal dado, $p$ es llamada prevalencia puntual.

Prevalencia puntual. La prevalencia puntual es la probabilidad de un individuo de una población de ser un caso en el momento $t$, y se calcula de la siguiente manera:

$p=\frac{\text { número total de casos existentes al momento } t}{\text { total de la población en el momento } t}(\times 10 \mathrm{n})$

La prevalencia de una enfermedad aumenta como consecuencia de una mayor duración de la enfermedad, la prolongación de la vida de los pacientes sin que éstos se curen, el aumento de casos nuevos, la inmigración de casos (o de susceptibles), la emigración de sanos y la mejoría de las posibilidades diagnósticas. La prevalencia de una enfermedad, por su parte, disminuye cuando es menor la duración de la enfermedad, existe una elevada tasa de letalidad, disminuyen los casos nuevos, hay inmigración de personas sanas, emigración de casos y aumento de la tasa de curación. En resumen, la prevalencia de una enfermedad depende de la incidencia y de la duración de la enfermedad.

Dado que la prevalencia depende de tantos factores no relacionados directamente con la causa de la enfermedad, los estudios de prevalencia no proporcionan pruebas claras de causalidad aunque a veces puedan sugerirla. Sin embargo, son útiles para valorar la necesidad de asistencia sanitaria, planificar los servicios de salud o estimar las necesidades asistenciales.

Anteriormente era común el cálculo de la llamada prevalencia de periodo (o lápsica), que buscaba identificar el número total de personas que presentaban la enfermedad o atributo a lo largo de un periodo determinado. No obstante, debido a las confusiones 
que origina, esta medida es cada vez menos empleada, y en materia de investigación es mejor no utilizarla.

\section{Incidencia}

En los estudios epidemiológicos en los que el propósito es la investigación causal o la evaluación de medidas preventivas, el interés está dirigido a la medición del flujo que se establece entre la salud y la enfermedad, es decir, a la aparición de casos nuevos. Como ya se mencionó anteriormente, la medida epidemiológica que mejor expresa este cambio de estado es la incidencia, la cual indica la frecuencia con que ocurren nuevos eventos. A diferencia de los estudios de prevalencia, los estudios de incidencia inician con poblaciones de susceptibles libres del evento en las cuales se observa la presentación de casos nuevos a lo largo de un periodo de seguimiento. De esta manera, los resultados no sólo indican el volumen final de casos nuevos aparecidos durante el seguimiento sino que permiten establecer relaciones de causa-efecto entre determinadas características de la población y enfermedades específicas. La incidencia de una enfermedad puede medirse de dos formas: mediante la tasa de incidencia (basada en el tiempo-persona) y mediante la incidencia acumulada (basada en el número de personas en riesgo). La tasa de incidencia (también denominada densidad de incidencia) expresa la ocurrencia de la enfermedad entre la población en relación con unidades de tiempo-persona, por lo que mide la velocidad de ocurrencia de la enfermedad. La incidencia acumulada, en cambio, expresa únicamente el volumen de casos nuevos ocurridos en una población durante un periodo, y mide la probabilidad de que un individuo desarrolle el evento en estudio. La incidencia acumulada, por esta razón, también es denominada riesgo.

Tasa de incidencia o densidad de incidencia. La tasa de incidencia (TI) es la principal medida de frecuencia de enfermedad y se define como "el potencial instantáneo de cambio en el estado de salud por unidad de tiempo, durante un periodo específico, en relación con el tamaño de la población susceptible en el mismo periodo". Para que una persona se considere expuesta al riesgo en el periodo de observación debe iniciar éste sin tener la enfermedad (el evento en estudio).

El cálculo del denominador de la TI se realiza sumando los tiempos libres de enfermedad de cada uno de los individuos que conforman el grupo y que permanecen en el estudio durante el periodo. Este número se mide generalmente en años, pero pueden ser meses, semanas o días, y se conoce como tiempo en riesgo o tiempo-persona.

El número de individuos que pasan del estado sano al estado enfermo durante cualquier periodo depende de tres factores: a) del tamaño de la población, b) de la amplitud del periodo de tiempo, y c) del poder patógeno de la enfermedad sobre la población. La tasa de incidencia mide este poder, y se obtiene dividiendo el número observado de casos entre el tiempo total en el que la población ha estado en riesgo, equivalente a la sumatoria de los periodos individuales en riesgo. Al sumar periodos de observación que pueden variar de uno a otro individuo y considerar sólo el tiempo total en riesgo la TI corrige el efecto de entrada y salida de individuos al grupo durante el periodo de seguimiento.

A menudo no es posible calcular exactamente la duración del tiempo-persona para los individuos que ya no están en riesgo, debido a que desarrollaron la enfermedad. No obstante, para este grupo el valor total del tiempo-persona en riesgo puede estimarse de manera aproximada -y generalmente satisfactoriamultiplicando el tamaño medio de la población por la duración del periodo de observación.

La TI no es una proporción -como la prevalencia y la incidencia acumulada- dado que el denominador expresa unidades de tiempo y, en consecuencia, mide casos por unidad de tiempo. Esto hace que la magnitud de la TI no pueda ser inferior a cero ni tenga límite superior. La fórmula general para el cálculo de la TI es la siguiente:

$$
\text { Tasa de incidencia }=\frac{\text { número de casos nuevos }}{\begin{array}{c}
\text { suma de todos los periodos libres } \\
\text { de la enfermedad durante el periodo }
\end{array}}
$$

Incidencia acumulada. La incidencia acumulada (IA) se puede definir como la probabilidad de desarrollar el evento, es decir, la proporción de individuos de una población que, en teoría, desarrollarían una enfermedad si todos sus miembros fuesen susceptibles a ella y ninguno falleciese a causa de otras enfermedades. También se ha definido simplemente como la probabilidad, o riesgo medio de los miembros de una población, de contraer una enfermedad en un periodo específico.

Las cifras obtenidas mediante el cálculo de la IA son relativamente fáciles de interpretar y proporcionan una medida sumamente útil para comparar los diferentes riesgos de distintas poblaciones. Para calcular la IA en el numerador se coloca el número de personas 
que desarrollan la enfermedad durante el periodo de estudio (llamados casos nuevos) y en el denominador el número de individuos libres de la enfermedad al comienzo del periodo y que, por tanto, estaban en riesgo de padecerla. La incidencia acumulada es una proporción y, por lo tanto, sus valores sólo pueden variar entre 0 y 1 . A diferencia de la tasa de incidencia la IA es adimensional. Su fórmula es la siguiente:

número de personas que contraen la enfermedad $\mathrm{IA}=\frac{\text { en un periodo determinado }}{\text { numero de personas libres de la enfermedad en la }}$ población expuesta al riesgo en el inicio del estudio

Como la duración del periodo de observación influye directamente sobre la IA su amplitud debe considerarse siempre que se interprete esta medida. Cuando los miembros de una población tienen diferentes periodos bajo riesgo -debido a que se incorporan o abandonan el grupo a lo largo del periodo de seguimiento- la IA no puede calcularse directamente.

\section{Medidas de asociación o de efecto}

Las medidas de asociación son indicadores epidemiológicos que evalúan la fuerza con la que una determinada enfermedad o evento de salud (que se presume como efecto) se asocia con un determinado factor (que se presume como su causa).

Epidemiológicamente, las medidas de asociación son comparaciones de incidencias: la incidencia de la enfermedad en las personas que se expusieron al factor estudiado (o incidencia entre los expuestos) contra la incidencia de la enfermedad en las personas que no se expusieron al factor estudiado (o incidencia entre los no expuestos). Estadísticamente, lo que estos indicadores miden es la magnitud de la diferencia observada. Debido a que las medidas de asociación establecen la fuerza con la que la exposición se asocia a la enfermedad, bajo ciertas circunstancias estas medidas permiten realizar inferencias causales, especialmente cuando se pueden evaluar mediante una función estadística. En este documento se abordará el cálculo de medidas de asociación para variables dicotómicas.

Las medidas de asociación más sólidas se calculan utilizando la incidencia, ya que esta medida de frecuencia nos permite establecer, sin ninguna duda, que el efecto (el evento o enfermedad) es posterior a la causa (la exposición). En estos casos, se dice, existe una correcta relación temporal entre la causa y el efecto. Empero, en los estudios en los que no existe suficiente información para calcular la incidencia (como las encuestas transversales y la mayoría de los estudios de casos y controles) no es posible calcular la incidencia. En estos casos puede estimarse la asociación entre el evento y la exposición al comparar las prevalencias a partir de la razón de prevalencias (RP) o de productos cruzados (RPC).

En general, hay dos tipos de medidas de asociación: las de diferencia (o de efecto absoluto) y las de razón (o de efecto relativo).

\section{Medidas de diferencia}

Como indica su nombre, estas medidas expresan la diferencia existente en una misma medida de frecuencia (idealmente la incidencia) entre dos poblaciones.

En general, las medidas de diferencia indican la contribución de un determinado factor en la producción de enfermedad entre los que están expuestos a él. $\mathrm{Su}$ uso se basa en la suposición de que tal factor es responsable de la aparición de la enfermedad y en la presunción de que, de no existir, los riesgos en ambos grupos serían iguales. Por este motivo, se dice que las medidas de diferencia indican el riesgo de enfermar que podría evitarse si se eliminara la exposición. Como sinónimo se emplea el término riesgo atribuible. Estas medidas se calculan de la siguiente manera:

$$
\text { Diferencia }=\mathrm{E}_{i}-\mathrm{E}_{o} \times 100
$$

donde,

$\mathrm{E}_{i}$ es la frecuencia de enfermar o morir de un grupo expuesto, $\mathrm{y}$

$\mathrm{E}_{0}$ es la frecuencia de enfermar o morir en el grupo no expuesto.

El resultado se interpreta de la siguiente forma:

Valor $=0$ indica no-asociación (valor nulo).

Valores $<0$ indica asociación negativa y puede tomar valores negativos hasta infinito.

Valores $>0$ indica asociación positiva y puede tomar valores positivos hasta infinito.

Debe señalarse que el término riesgo atribuible carece de justificación cuando no existe una relación causa-efecto entre la exposición y la enfermedad. No obstante, como la diferencia de incidencias -ya sea diferencia de tasas de incidencia (DTI) o diferencia de riesgos (DR)- puede llegar a indicar diferencias verdaderamente atribuibles a la exposición, estas medidas se siguen usando para estimar la magnitud de proble- 
mas de salud pública, aunque ya casi nunca se usan en investigación.

La diferencia de prevalencia (DP), usada en estudios transversales, puede ser en algunas condiciones un estimador aceptable de la diferencia de incidencia, pero sus resultados sólo indican asociación y no causalidad.

\section{Medidas de razón}

Estas medidas también cuantifican las discrepancias en la ocurrencia de enfermedad en grupos que difieren en la presencia o no de cierta característica. Como se señaló antes, una razón puede calcularse tanto para dos eventos en una misma población como para un solo evento en dos poblaciones. Las razones que con mayor frecuencia se calculan son del segundo tipo, y se obtienen con la siguiente fórmula:

Razón $=\frac{\text { medida de frecuencia en un grupo expuesto }\left(\mathrm{E}_{\mathrm{i}}\right)}{\text { medida de frecuencia de un grupo no expuesto }\left(\mathrm{E}_{\mathrm{o}}\right)}$

La razón representa cuántas veces más (o menos) ocurrirá el evento en el grupo expuesto al factor, comparado con el grupo no expuesto. El resultado se interpreta de la siguiente forma:

Valor =1 indica ausencia de asociación, no-asociación o valor nulo.

Valores $<1$ indica asociación negativa, factor protector. Valores $>1$ indica asociación positiva, factor de riesgo.

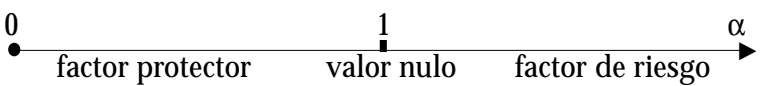

La interpretación de estas medidas se basa en el hecho de que si se dividen dos cantidades entre sí y el resultado es 1, estas cantidades son necesariamente iguales, y tener o no la característica estudiada es lo mismo, pues ello no afecta la frecuencia de enfermedad. Cuando, en cambio, la razón es mayor de 1, el factor se encuentra asociado positivamente con el riesgo de enfermar y la probabilidad de contraer el padecimiento será mayor entre los expuestos. Si el resultado es menor de 1, el factor protege a los sujetos expuestos contra esa enfermedad.

Conforme el resultado se aleja más de la unidad, la asociación entre el factor y la enfermedad es más fuerte. Un valor de 4 indica que el riesgo de enfermar entre los expuestos es cuatro veces mayor que entre los no expuestos. Asimismo, un valor de 0.25 indicaría que el riesgo de enfermar entre los expuestos es cuatro veces menor que entre los no expuestos.
La incidencia y la mortalidad son las medidas de frecuencia más empleadas en la construcción de las medidas de razón. Con la densidad de incidencia se obtiene la razón de densidad de incidencia (RDI), y con la incidencia acumulada se obtiene la razón de incidencia acumulada (RIA) también llamado riesgo relativo (RR). Ambas medidas -que se obtienen en estudios de cohorte- permiten asumir inferencia etiológica, ya que siempre implican la posibilidad de establecer adecuadamente una relación de temporalidad causal.

\section{Razón de densidad de incidencia}

Esta medida es útil para identificar la velocidad con la que se pasa del estado sano al de enfermo según se esté expuesto o no a determinado factor.

Razón de incidencia acumulada o riesgo relativo

Compara el riesgo de enfermar del grupo de expuestos $\left(\mathrm{IA}_{i}\right)$ con el riesgo de enfermar del grupo de no expuestos (IA o). Es útil si lo que se desea es conocer la probabilidad de padecer la enfermedad en función de la exposición, y es la medida que mejor refleja su asociación.

$\mathrm{RR}=\frac{\mathrm{IA}_{i}}{\mathrm{IA}_{o}}=\frac{\mathrm{a} / \mathrm{n}_{i}}{\mathrm{c} / \mathrm{n}_{o}}$

donde,

$\mathrm{IA}_{i}$ es la incidencia acumulada o riesgo de enfermar entre los expuestos, $y$

$\mathrm{IA}_{0}$ es la incidencia acumulada o riesgo de enfermar entre los no expuestos (para observar gráficamente la ubicación de las celdas a, $\mathrm{c}, \mathrm{n}_{i} \mathrm{y}_{0^{\prime}}$ véase la tabla de $2 \times 2$ ).

\section{Razón de prevalencias}

La razón de prevalencias (RP) se utiliza en los estudios transversales y se calcula de forma similar a la estimación del RR en los estudios de cohorte. Si la duración del evento que se estudia es igual para expuestos y no expuestos, la RP puede ser buen estimador de la velocidad con la que se pasa del estado sano al de enfermo, pero, en general, esta medida subestima la RDI.

Razón de productos cruzados

La razón de productos cruzados (RPC u OR) se estima en los estudios de casos y controles -donde los su- 
jetos son elegidos según la presencia o ausencia de enfermedad, desconociéndose el volumen de la población de donde provienen- por lo que no es posible calcular la incidencia de la enfermedad. La RPC también se conoce con los términos en inglés odds ratio (OR) y relative odds, y en español como razón de momios (RM), razón de ventaja y razón de disparidad. La RM es un buen estimador de la RDI, sobre todo cuando los controles son representativos de la población de la que han sido seleccionados los casos. La RM también puede ser un buen estimador del RR. Esta medida se calcula obteniendo el cociente de los productos cruzados de una tabla tetracórica:

$\mathrm{RPC}=\frac{\mathrm{a} / \mathrm{c}}{\mathrm{b} / \mathrm{d}}=\frac{\mathrm{ad}}{\mathrm{bc}}$

donde,

\begin{tabular}{|ccc|c|c|}
\cline { 2 - 5 } & \multicolumn{2}{|c|}{ casos } & controles & \\
\cline { 2 - 5 } Exposición & presente & $\mathrm{a}$ & $\mathrm{b}$ & $\begin{array}{c}\text { Total de } \\
\text { expuestos }\left(\mathrm{n}_{i}\right)\end{array}$ \\
\cline { 2 - 5 } & ausente & $\mathrm{c}$ & $\mathrm{d}$ & $\begin{array}{c}\text { Total de no } \\
\text { expuestos }\left(\mathrm{n}_{o}\right)\end{array}$ \\
\cline { 2 - 5 } & Total $\left(\mathrm{m}_{i}\right)$ & Total $\left(\mathrm{m}_{\mathrm{o}}\right)$ & $\begin{array}{c}\text { Total de } \\
\text { sujetos }(\mathrm{n})\end{array}$ \\
\hline
\end{tabular}

$\mathrm{Al}$ igual que en las medidas anteriores, esta fórmula expresa el caso más sencillo, cuando la exposición y la enfermedad se reportan simplemente como presentes o ausentes.

El resultado se interpreta de la misma forma que en el resto de las medidas de razón. Cuando la OR tiene un valor de 1 (o nulo), el comportamiento del factor es indiferente; si el valor es superior a 1 , el factor puede considerarse como de riesgo, y si es inferior a 1 es valorado como factor protector.

\section{Medidas de impacto potencial}

La razón de densidad de incidencia, el riesgo relativo y la razón de momios describen la asociación entre la exposición y el evento en términos de la magnitud de la fuerza de la asociación entre estos, información que es muy importante cuando evaluamos la existencia de asociaciones causales. Sin embargo, estas medidas no se pueden traducir fácilmente en el contexto de la salud de la población. ¿Qué tan importante es una exposición? ¿Qué proporción de las enfermedades se pueden atribuir a esta variable? Para poder estimar el efecto de cierta exposición en la población en estudio o en la población blanco se requiere esti- mar otro tipo de medidas, conocidas como medidas de impacto.

Las principales medidas de impacto potencial son el riesgo atribuible (o fracción etiológica), que se estima cuando el factor de exposición produce un incremento en el riesgo $(R R>1)$, y la fracción prevenible, relacionada con factores que producen una disminución en el riesgo $(R R<1)$.

\section{Riesgo atribuible}

Anteriormente era muy frecuente el uso del termino fracción etiológica para referirse a este indicador; sin embargo, actualmente se recomienda utilizarlo únicamente para referirse a relaciones causales bien demostradas. El termino que se usa con mayor frecuencia y que es más conservador es el riesgo atribuible proporcional. Para esta ultima medida se han derivado dos dimensiones, el Riesgo Atribuible Proporcional en el grupo Expuesto $\left(\mathrm{RAP}_{\mathrm{Exp}}\right)$ y el Riesgo Atribuible Proporcional en la Población blanco (RAPP). Ambas medidas son proporciones, por lo que toman valores entre cero y uno e indican la importancia relativa de la exposición al factor en estudio con relación al total de

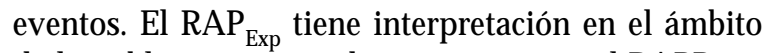
de la población en estudio, mientras que el RAPP expresa la importancia en el ámbito poblacional, o población blanco.

El $\mathrm{RAP}_{\text {Exp }}$ estima la proporción de eventos en el grupo expuesto que se pueden atribuir a la presencia del factor de exposición. En otras palabras, refleja el efecto que se podría esperar en el grupo expuesto de la población en estudio si se eliminara el factor de riesgo en cuestión. El RAP $\mathrm{Exp}_{\text {p }}$ se puede calcular utilizando la siguiente fórmula:

RAPexp $=\frac{\text { DIE-DINE }}{\text { DIE }}=\frac{\text { RDI- } 1}{\text { RDI }}$

donde

$\mathrm{DI}_{\mathrm{E}}=$ Densidad de incidencia en expuestos,

$\mathrm{DI}_{\mathrm{NE}}=$ Densidad de incidencia en no expuestos, $\mathrm{y}$

RDI= Razón de densidad de incidencia

El $\mathrm{RAP}_{\text {Exp }}$ se puede estimar también en estudios donde la medida de frecuencia es la incidencia acumulada, utilizando el riesgo relativo. Además, dado que la razón de momios es un buen estimador de la RDI, el RAPexp también se puede estimar en los estudios de casos y controles, utilizando la siguiente fórmula: 
$\mathrm{RAPexp}=\frac{\mathrm{RM}-1}{\mathrm{RM}}$

Para ilustrar su interpretación y cálculo supongamos que se desea estimar el $\mathrm{RAP}_{\text {Exp }}$ de los resultados derivados de un estudio de casos y controles sobre tabaquismo y cáncer pulmonar. En el mencionado estudio se documenta un asociación entre el riesgo de cáncer de pulmón y el tabaquismo (RM) de 12.5. El $\mathrm{RAP}_{\text {Exp }}$ se podría estimar dividiendo 12.5 menos 1 entre 12.5, lo que daría un $\operatorname{RAP}_{\text {Exp }}$ de 0.92 (o $92 \%$ ), lo que indicaría que el $92 \%$ de los casos de cáncer pulmonar en el grupo expuesto al tabaco podrían atribuirse a esta exposición. Esto significa que el $\mathrm{RAP}_{\text {Exp }}$ indica el porcentaje de casos en el grupo expuesto que se podría prevenir si se eliminara la exposición, asumiendo que la exposición es la única causa del evento y que el resto de las causas de cáncer de pulmón se distribuyen de igual manera entre los fumadores (grupo expuesto) y los no fumadores (grupo no expuesto), como se indica en la figura 1. Para el ejemplo anterior indicaría que se podrían prevenir cerca del $92 \%$ de los casos de cáncer de pulmón que ocurren en el grupo de fumadores.

El RAPP se puede considerar como una proyec-

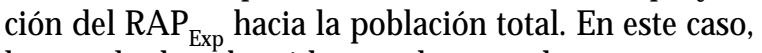
los resultados obtenidos en el grupo de expuestos se extrapolan hacia la población blanco estimando el impacto de la exposición a nivel poblacional. Siguiendo el ejemplo anterior, la estimación del RAPP nos indicaría cuántos casos de cáncer de pulmón en la población total son atribuibles al tabaco o se podrían evitar supo-

\section{Fracción atribuible a la exposición en expuestos}

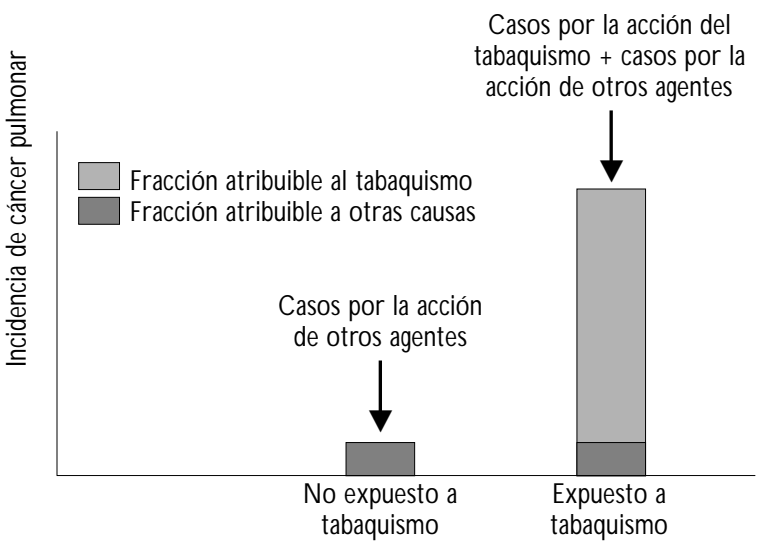

Figura 1. Representación hipotética de Un estudio DE COHORTE PARA EVALUAR EL EFECTO DEL TABAQUISMO SOBRE EL RIESGO DE DESARROLLAR CÁNCER DE PULMÓN niendo que se eliminara el tabaquismo en la población general. EL RAPP se estima ponderando el $\mathrm{RAP}_{\text {Exp }}$ de acuerdo con la proporción de sujetos expuestos en la población blanco. El RAPP se puede estimar utilizando la siguiente formula:

RAPP $=\frac{\operatorname{Pe}(\text { RDI }-1)}{\operatorname{Pe}(\text { RDI-1)+1 }}$

$\mathrm{Al}$ igual que en el caso anterior, el RAPP se puede estimar para estudios de cohorte, donde se estima la incidencia acumulada, o en estudios de casos y controles, donde se estima la razón de momios. En este ultimo caso, se puede utilizar la prevalencia de exposición en los controles para estimar la prevalencia en la población blanco o población de referencia. En el estudio antes mencionado sobre tabaquismo y cáncer pulmonar se observo una prevalencia del 28.5 de tabaquismo en el grupo control. Dado que la serie de controles se puede considerar como representativa de la población base, en este estudio se podría estimar directamente el RAPP, lo que daría una fracción de 0.76 . Esta ultima cifra indicaría que, en la población blanco, el $76 \%$ de los casos de cáncer pulmonar pueden ser atribuidos al tabaquismo, asumiendo que el tabaquismo es su única causa.

Mediante el calculo del RAP $_{\text {Exp }}$ y del RAPP es posible identificar diversos escenarios:

a) Con un RR alto y una prevalencia de expuestos alta, la reducción del riesgo de enfermedad puede considerarse como de alto impacto.

b) Cuando el RR es bajo y la prevalencia de expuestos es alta, la supresión del factor de riesgo posee un impacto moderado, pero notable entre los expuestos.

c) Cuando el RR es alto pero la prevalencia de expuestos es baja, la eliminación del factor de riesgo tiene un impacto relativamente bajo tanto entre la población blanco como entre los expuestos, $\mathrm{y}$

d) Cuando el RR es bajo y la prevalencia de expuestos también es baja, la eliminación del factor de riesgo no es una prioridad en salud pública, ya que su impacto en la población blanco y en los expuestos sería irrelevante.

\section{Fracción prevenible}

Esta medida se aplica cuando a partir de las medidas de asociación se obtienen factores protectores o negativos $(\mathrm{RR}<1)$. También existen dos modalidades: fracción prevenible poblacional y fracción prevenible entre expuestos. 
La fracción prevenible poblacional es la proporción de todos los casos nuevos que potencialmente podrían haber ocurrido entre la población general en un determinado periodo en ausencia de una exposición protectora específica; o bien, es la proporción de casos potenciales que serían realmente prevenibles o evitados si existiera la exposición entre la población.

Finalmente, la fracción prevenible para los expuestos es la proporción de casos nuevos entre los expuestos que potencialmente podría haber ocurrido en un determinado periodo en ausencia de una exposición particular. Es decir, es la proporción de casos expuestos potenciales que realmente se evitarían si la población se expusiera al factor protector.

\section{Bibliografía}

1. MacMahon B, Pugh TF. Epidemiology. Principles and methods. Boston: Little Brown \& C 0., 1970.

2. Kleimbaum DG, Kupper LL, Morgenstern H. Epidemiologic research. N ey York (N Y): Van N ostrand Reinhold C 0., 1982.

3. Rothman JK. Modern epidemiology. Boston: Little Brown \& C 0., 1986. 4. A hlbom A, Norell S. Fundamentos de epidemiología. Madrid: Siglo XXI Editores 1987.

5. Gordis L. Epidemiology. Philadelphia:W .B. Saunders C 0. 1995.

6. Jenicek M. Epidemiología: la lógica de la medicina moderna, Barcelona: Masson, 1996.

7. Martínez N F,A ntó JM, C astellanos PL, G ili M, Marset P, N avarro V. Salud pública. Madrid: MCG raw-Hill-Interamericana, 1998. 